\title{
Entropy in hadron-hadron collisions
}

\author{
K. Fiałkowski円, R. Witß \\ M. Smoluchowski Institute of Physics \\ Jagellonian University \\ 30-059 Kraków, ul.Reymonta 4, Poland
}

\begin{abstract}
We analyse the entropy properties in the proton - proton $1800 \mathrm{GeV}$ events from the PYTHIA/JETSET Monte Carlo generator following a recent proposal concerning the measurement of entropy in multiparticle systems. The dependence on the number of bins and on the size of the phase-space region is investigated. Our results may serve as a reference sample for experimental data from hadron-hadron and heavy ion collisions.
\end{abstract}

PACS: $13.85 . \mathrm{Hd}$

Keywords: hadron-hadron collisions, entropy, Monte Carlo

13 July, 2000

\footnotetext{
${ }^{1}$ e-mail address: uffialko@thrisc.if.uj.edu.pl

${ }^{2}$ e-mail address: wit@thrisc.if.uj.edu.pl
} 


\section{Introduction}

In a recent series of papers Ref. [1], [2], [3] a specific proposal was presented for entropy measurement of multiparticle systems created in high-energy collisions. The proposal should be important for the analysis of the forthcoming RHIC experiments where it may help in the separation of a possible signal from quark-gluon plasma (QGP). However, this proposal is not merely restricted to systems with a very large number of particles. Applying it to other multiparticle systems, e.g. originating from hadron-hadron collisions, one may get useful reference data for the discussion of the thermodynamic equilibrium and other properties of such systems.

In this note we use the PYTHIA/JETSET event generator [4] to create samples of multiparticle states and analyse them according to the proposal mentioned above. In the next section we remind shortly the procedure presented in Ref. [3] and specify the process and variables used in the analysis. The results are presented in the third section. The last section contains a discussion of the results including some conclusions and perspectives.

\section{Procedure and variables}

We generate samples of $10^{5}$ or $10^{6}$ events of $p p$ collisions at $1800 \mathrm{GeV} \mathrm{CM}$ energy, the highest energy available yet for hadron-hadron collisions. This ensures a relatively high particle density leaving the possibility for comparison with experimental data. For each event the phase space region of a few units in rapidity (in the central region) and $p_{T}^{2}$ restricted to less than $0.4 \mathrm{GeV}^{2} / \mathrm{c}^{2}$ is used.

To calculate the entropy we "discretize" each event. For definiteness we are using bins in $p_{T}^{2}$; binning in rapidity leads to similar results. The $p_{T}^{2}$ range is divided into $M$ bins, and the number of particles in each bin $m_{i}, i=1, \ldots, M$ is recorded. Now it is possible to calculate the Shannon entropy from the standard definition

$$
S=-\sum_{j} p_{j} \log p_{j}
$$

where $p_{j}$ denotes the probability to obtain any specific configuration of numbers $\left\{m_{i}\right\}$. Obviously, $p_{j}=n_{j} / N$ where $n_{j}$ is the number of events providing such configuration and $N$ is the global number of events.

However, in the proposal [3] the calculation of entropy is performed in a different way for the reasons to be discussed later. First, one calculates total numbers of observed coincidences of $k$ configurations $N_{k}$

$$
N_{k}=\sum_{j} n_{j}\left(n_{j}-1\right) \ldots\left(n_{j}-k+1\right) .
$$

Then the coincidence probability of $k$ configurations is given by

$$
C_{k}=\frac{N_{k}}{N(N-1) \ldots(N-k+1)} .
$$


These probabilities are used to calculate Renyi entropies as

$$
H_{k}=-\frac{\log C_{k}}{k-1}
$$

instead of calculating them directly from the following definition

$$
H_{k}=\sum_{j}\left(p_{j}\right)^{k} .
$$

We will comment later on the Renyi entropy values obtained by these two methods.

The Shannon entropy is formally equal to the limit of Renyi entropies $H_{k}$ as $k \rightarrow 1$ and can be obtained by extrapolation. Obviously $N_{1}=N, C_{1}=1$, and this extrapolation cannot be done just by putting $k=1$ in formula (耳). It was suggested [2] to use for the extrapolation a formula

$$
H_{k}=a \frac{\log k}{k-1}+a_{0}+a_{1}(k-1)+a_{2}(k-1)^{2}+\ldots,
$$

where the number of terms is determined by the number of measured Renyi entropies. Usually it is enough to use $H_{k}$ for $k=2,3,4$. Other extrapolations can also be used, e.g.

$$
H_{k}=a_{0}+a_{1} k^{-1}+a_{2} k^{-2}+\ldots
$$

and should be compared with the one presented here to estimate the extrapolation accuracy. We comment on this later on.

It was suggested that for a system close to equilibrium and small bins the entropy should grow logarithmically with the number of bins

$$
H_{k}(l M)=H_{k}(M)+\log l \Rightarrow S(l M)=S(M)+\log l .
$$

Another expected feature is additivity: for entropies measured in a phase-space region $R$, which is the sum of two regions $R_{1}$ and $R_{2}$, we should observe

$$
H_{k}(R)=H_{k}\left(R_{1}\right)+H_{k}\left(R_{2}\right) \Rightarrow S(R)=S\left(R_{1}\right)+S\left(R_{2}\right) .
$$

We check these features by choosing different numbers of bins in $p_{T}^{2}$ and different ranges of rapidity.

As we have seen, for all our calculations we need $n_{j}$, the numbers of events providing specific configurations of numbers of particles in bins $m_{i}$. It may be difficult to record all $n_{j}$ since the number of different possible configurations grows quickly with the number of bins and particles. If for each of $\mathrm{M}$ bins the number of particles may change within an interval length $L$ the number of a priori possible configurations is $L^{M}$. This is a rather big number even for moderate values of $L$ and $M$.

However, we need to know only the values of $n_{j}$ and not the form of the configurations corresponding to each value of $n_{j}$. Therefore it is really not necessary to make big computer memory reservations. Instead of initializing a big matrix with all elements equal to zero and filling it gradually with generated events, we define $n_{j}$ only for those configurations which actually appear in generated events.

Still, the registration of all $n_{j}$-s and consecutive calculations may be quite time consuming. In our case we have found that the computing time may become prohibitive for $10^{6}$ events and $9 p_{T}^{2}$ bins. Therefore it is important to find the lowest possible number of events for which the results become stable. 


\section{Results}

To start discussing any results one should know how reliable they are and what is their uncertainty. Therefore we estimated first the dependence of entropy values on the generated number of events. We checked that the results for $10^{5}$ and $10^{6}$ event samples differ most for largest number of bins and largest rapidity range $\Delta y$. We show this effect in Tab.1, where the values of Renyi and Shannon entropies are presented for 9 bins in $p_{T}^{2}$ and different values of $\Delta y$ for these two samples of events. Shannon entropy is calculated by extrapolation and from the direct definition (1). For smaller number of bins all differences are smaller, but the pattern is the same.

TAB.1. Entropy values for 9 bins in $p_{T}^{2}$ for changing $\Delta y$ from $10^{6}\left(10^{5}\right)$ events.

\begin{tabular}{||c|c|c|c|c|c|}
\hline \hline & \multicolumn{5}{|c|}{$\Delta y$} \\
\hline \hline Entropy & 1 & 2 & 3 & 4 & 6 \\
\hline \hline$H_{4}$ & $3.67(3.66)$ & $5.84(5.84)$ & $7.47(7.51)$ & $8.85(8.90)$ & $11.03(11.07)$ \\
\hline$H_{3}$ & $3.99(3.98)$ & $6.27(6.28)$ & $8.00(8.03)$ & $9.43(9.46)$ & $11.63(11.66)$ \\
\hline$H_{2}$ & $4.68(4.68)$ & $7.19(7.20)$ & $9.03(9.04)$ & $10.51(10.52)$ & $12.69(12.70)$ \\
\hline$S^{e x}$ & $6.44(6.45)$ & $9.47(9.47)$ & $11.53(11.51)$ & $13.03(13.04)$ & $15.07(15.07)$ \\
\hline$S^{d f}$ & $6.76(6.64)$ & $9.46(9.04)$ & $11.06(10.23)$ & $12.07(10.88)$ & $13.12(11.36)$ \\
\hline \hline
\end{tabular}

The same results are shown in Fig.1. However, for the sake of transparency only the values of Shannon entropies and second Renyi entropies are presented.

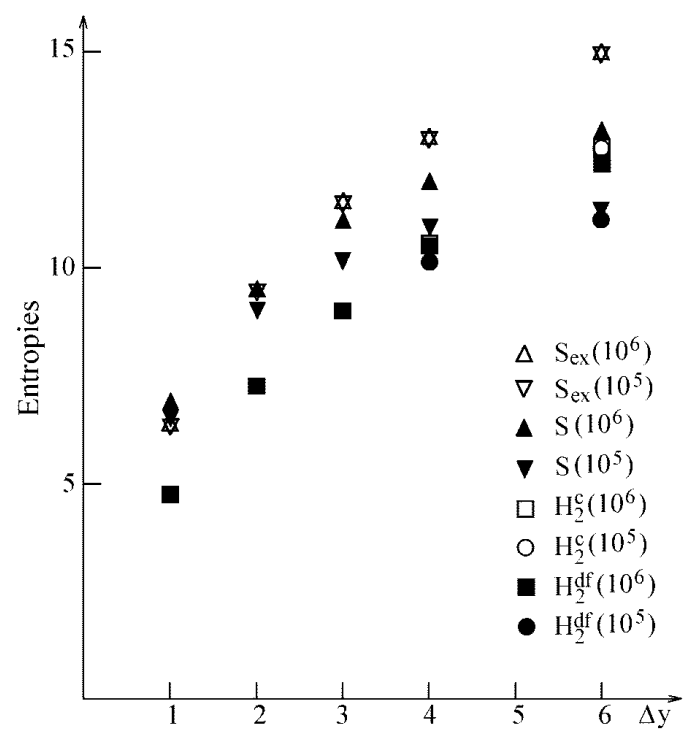

Figure 1: Shannon entropy calculated for 9 bins in $p_{T}^{2}$ for $10^{5}$ events (inverted triangles) and for $10^{6}$ events (triangles) from the definition (四) (black symbols) and by extrapolation of formula (6) (open symbols) as a function of rapidity range $\Delta y$. Second Renyi entropy is also shown for $10^{5}$ events (circles) and $10^{6}$ events (squares) calculated from definition (5) (black symbols) and from the coincidence probabilities (田) (open symbols). 
We see that for narrow ranges of rapidity $\Delta y$ the values of entropy for $10^{5}$ and $10^{6}$ events are very similar and the differences grow with $\Delta y$. The most striking effect is that these differences stay always small when Shannon entropy $S$ is calculated by the extrapolation from Renyi entropies $H_{k}$ to $k=1$ according to formula (6), whereas the values calculated directly from the definition (1) differ really strongly for two samples at widest rapidity ranges. In fact, the entropy values calculated for $10^{5}$ events from the definition (1) seem to saturate at the level of 11.5 , which is close to $\log 10^{5}$.

This confirms that the method proposed in [1], [2] is indeed much better than the direct measurment of Shannon entropy (unless the number of particles in the bin is too small). For this method it is possible to calculate the Shannon entropy reliably even for modest samples of events. In the following we show only the values obtained by the extrapolation procedure. We checked that the results for the second extrapolation (7) are the same within $2 \%$ accuracy.

For the second (and further) Renyi entropies the results for two samples never differ too much. The difference is still smaller if they are calculated by the advised procedure from coincidence probabilities (4) and not directly from the definition (5). Further results shown use always this procedure.

Before testing the additivity of entropy (relation(8)) we perform a simple exercise. Since it was suggested that additivity may be broken by correlation effects, we checked if the short-range correlations are relevant. To this purpose we calculated the entropies for the same number of bins in $p_{T}^{2}$ using the rapidity range $\Delta y=2$ centered at $C M$ rapidity zero in "one piece" $(-1<y<1)$ and in two intervals of width 1 separated by a gap of two units $(-2<y<-1$ and $1<y<2)$. As seen in Fig.2, the results are barely distinguishable, which shows that the short range correlation effects are negligible for our discussion.

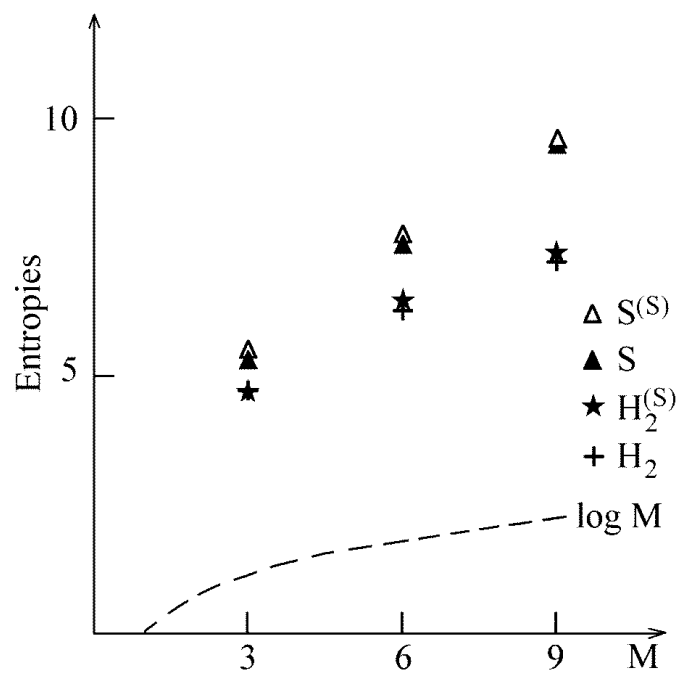

Figure 2: Shannon entropy for compact (black triangles) and separated (open triangles) phase space regions in rapidity of two units width as a function of number of bins $M$. Second Renyi entropy is also shown (crosses and stars, respectively). 
The dependence of the entropy on the number of bins seen in this figure seems to be significantly stronger than that predicted by eq. (7): the $\log M$ curve is shown on the bottom of the Fig.2 for comparison.

The irrelevance of short-range correlations allows us to test additivity simply by plotting the dependence of entropy values on the width of rapidity range $\Delta y$. Entropy should be proportional to $\Delta y$ (at least in the central region, where the rapidity distribution is approximately flat; our values of $\Delta y$ correspond always to this region). We check it for two choices of bins: of equal width in $p_{T}^{2}$ (as in all other figures) and for the same number of bins, the same range of $p_{T}^{2}$, but bin sizes defined by a requirement of approximately equal average multiplicities. The results are shown in Fig. 3 for 6 bins; for other numbers of bins the pattern is the same.

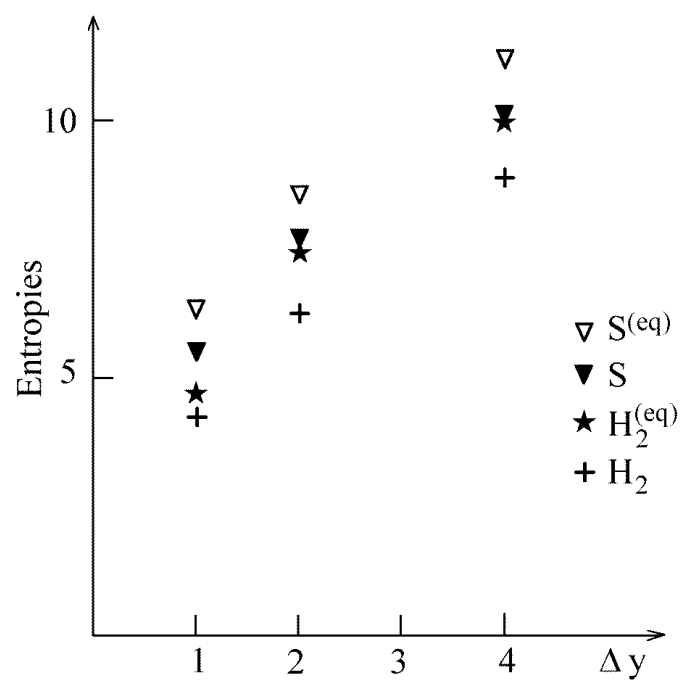

Figure 3: Shannon entropy for 6 bins in $p_{T}^{2}$ of equal width (black triangles) and of equal multiplicity (open triangles) as a function of rapidity range $\Delta y$. Second Renyi entropy is also shown (crosses and stars, respectively).

We see that the results for two binning procedures differ just by shifting the entropy values; the dependence on rapidity range is the same and in both cases it is definitely weaker than linear. Thus there is no additivity in the sense of eq. (8), which turn suggests that there is no thermal equilibrium in the process under investigation.

Finally, we test the dependence of entropies on the number of bins when the average multiplicities per bin remain unchanged (i.e. we increase the rapidity range proportionally to the number of bins in $p_{T}^{2}$ keeping thus the "bin volume" $\Delta V=\Delta p_{T}^{2} \cdot \Delta y$ constant). The results are shown in Fig.4. As one sees, the dependence is in this case approximately linear.

\section{Conclusions and outlook}

We have calculated Shannon entropies for the final states from the $p p$ collisions at $1800 \mathrm{GeV}$ CM energy using the PYTHIA/JETSET event generator. We found that for the proce- 


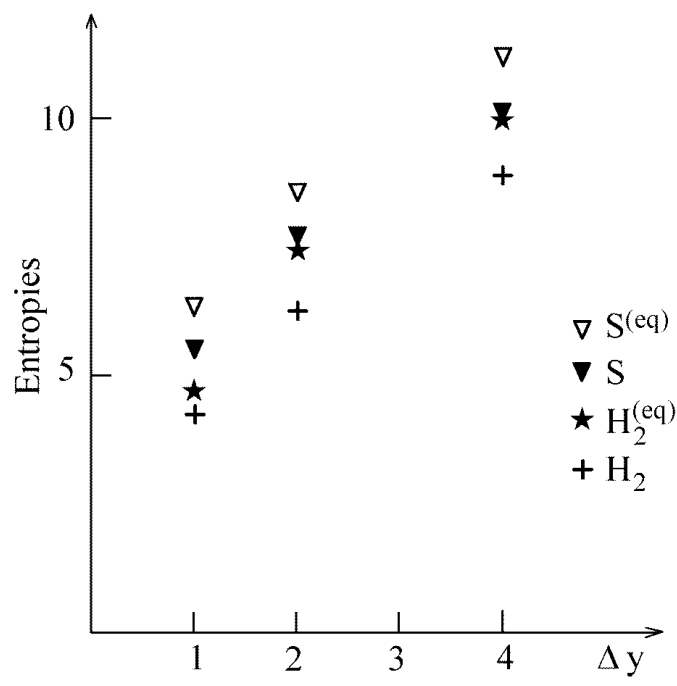

Figure 4: Shannon entropy for equal width bins in $p_{T}^{2}$ of constant bin volume $\Delta V=\Delta y \cdot \Delta p_{T}^{2}$ as a function of number of bins $M$. Black (open) triangles are for $\Delta V=0.133 \mathrm{GeV}^{2}\left(0.267 \mathrm{GeV}^{2}\right)$. Second Renyi entropy is also shown (crosses and stars, respectively).

dure extrapolating Renyi entropies it is enough to generate $10^{5}$ events to get numerically stable results.

We have tested the conjecture that entropy is additive, i.e. that entropy measured in a phase-space region $R$ which is the sum of two regions $R_{1}$ and $R_{2}$ is just a sum of entropies measured in these two regions. Our results do not confirm this conjecture; the increase of entropy with the size of the phase-space region is slower than linear. This is may be regarded as the effect of correlations. We show that it is dominated by long range correlations; the results for two adjacent regions and separated regions are almost the same.

We have also investigated the dependence of entropy on the number of bins. It seems to be stronger than the expected logarithmic, perhaps due to a small number of bins. If we keep the average multiplicity per bin unchanged and increase both the number of bins and the size of the relevant phase-space region, we find an approximately linear increase of entropy.

Our investigation shows that it is feasible to perform a program proposed in Refs. [1], [2] and [3] for experimental data. The procedure will be the same as for our samples of generated events. We have shown that for hadron-hadron collisions the results are stable already for $10^{5}$ events. Obviously, for the high multiplicity heavy ion collisions one should take much smaller bins to have comparable multiplicities; otherwise one should check again the stability conditions.

The results presented above may also serve as a reference sample for the experimental data resulting both from hadron-hadron and heavy ion collisions. Since the used generator does not assume any thermodynamical equilibrium, the observed similarities and differences may help in the discussion concerning the presence of equilibrium in data.

It would be useful to perform a similar analysis for different choices of variables and for 
different generators, in particular for those which are dedicated for heavy ion collisions.

\section{Acknowledgements}

We would like to thank A. Białas and W. Czyż for reading the manuscript. The financial support of KBN grants \# 2 P03B 08614 and \# 2P03B 01015 is gratefully acknowledged. One of us (RW) is grateful for a partial financial support by the KBN grant \# 2 P03B 01917 and the other one (KF) for the support of FNP (subsydium FNP 1/99 granted to A. Białas).

\section{References}

[1] A. Białas, W. Czyż and J. Wosiek, Acta Phys. Polon. 30, 107 (1999).

[2] A. Białas and W. Czyż, Phys. Rev. D61, 074021 (2000).

[3] A. Białas and W. Czyż, Acta Phys. Polon. 31, 687 (2000).

[4] T. Sjöstrand and M. Bengtsson, Comp. Phys. Comm. 43, 367 (1987);

T. Sjöstrand, Comp. Phys. Comm. 82, 74 (1994). 